\title{
Application of Nonlinear Dynamics Tools for Diagnosis of Cracked Rotor Vibration Signatures
}

\author{
Jerzy T. Sawicki ${ }^{*}$ Xi Wu* , Andrew L. Gyekenyesi ${ }^{* *}$, George Y. Baaklini ${ }^{\dagger}$ \\ *Cleveland State University, Dept. of Mechanical Engineering, Cleveland, OH 44115; \\ ** OAI/NASA Glenn Research Center, Cleveland, OH 44135; \\ ${ }^{\dagger}$ NASA Glenn Research Center, Cleveland, $\mathrm{OH} 44135$
}

\begin{abstract}
The nonlinear model of the cracked Jeffcott rotor is investigated, with the particular focus on study of rotor's vibrational response using tools of nonlinear dynamics. The considered model accounts for nonlinear behavior of the crack and coupling between lateral and torsional modes of vibrations. Load torque is applied to the rotor which is laterally loaded with a constant radial force (gravity force) and unbalance excitation. The co-existence of frequencies of lateral modes in the frequency spectra of torsional mode are characteristics of the coupling response of lateral and torsional vibrations. When only the lateral excitations are applied, vibration amplitude bifurcation plot with the shaft speed as a control parameter, demonstrates some speed ranges for which vibrations of the rotor dramatically increase. Furthermore, the torsional response amplitude at the same speed ranges also increases and chaotic behavior can be observed due to the lateral excitations. These phenomena cannot be observed for pure lateral vibration response with the torsionally rigid rotor assumption.
\end{abstract}

Keywords: Jeffcott rotor, crack, nonlinear dynamics, vibration signatures, bifurcation diagram, detection.

\section{INTRODUCTION}

The tendency to higher speeds in turbomachinery results in design of more flexible shafts, which spin at speeds above several of their natural frequencies. Due to stress concentration and high spin speed, rotor dynamic system is more prone to cracks which propagate faster due to low-cycle fatigue loading. In many rotating machinery, the lateral natural frequencies are far much lower than the lowest torsional natural frequency. That's why most of the condition monitoring methods is based on lateral vibrations. Without proper transducer, it's very difficult to detect torsional vibrations even when their amplitudes are close to dangerous level. However, in order to capture the whole picture of the cracked dynamic system, and develop a high efficiency on on-line condition monitoring method, the lateral and torsional coupling must be taken into consideration, because torsional excitation generate not only torsional responses, but also cause lateral vibrations due to the mechanism of torsional and lateral coupling.

Finite Element Method is basically a linear approach, which ignores the lateral and torsional coupling mechanism of the uncracked rotor. It is only due to the crack, which introduces nonlinearity and produces coupling terms of cracked rotor in the FEM-based model. But, the coupling does exist in an uncracked rotor dynamic system. Tondl [1], Cohen and Porat [2] and Bernasconi [3] concluded that typical lateral excitations, such as unbalance, may result in both lateral and torsional responses of uncracked rotor. Furthermore, Muszynska et al. [4] and Bently et al. [5] discuss rotor coupled lateral and torsional vibrations due to unbalance, as well as due to shaft anisotropy and radial constant preload force. Their experimental results exhibited the existence of significant torsional vibrations, due to coupling with the lateral modes. Sawicki et al. [6,7] studied transient response of the cracked rotor, including the stalling effect under the constant driving torque.

\section{EQUATION OF MOTION OF A CRACKED JEFFCOT ROTOR}

A cracked Jeffcott rotor with lateral and angular (torsional) degrees of freedom is considered. The rotor is subjected to a constant radial load (gravity force), rotating unbalance force, and the externally applied torque. The coupled torsional- 
flexural vibrations are studied for a rotor with a breathing crack model. The sectioned view of the cracked rotor, in both inertial and rotating coordinates, is shown in Fig. 1. The stiffness of the uncracked rotor system is symmetric (isotropic), and the damping due to the air resistance effect is assumed to be viscous.

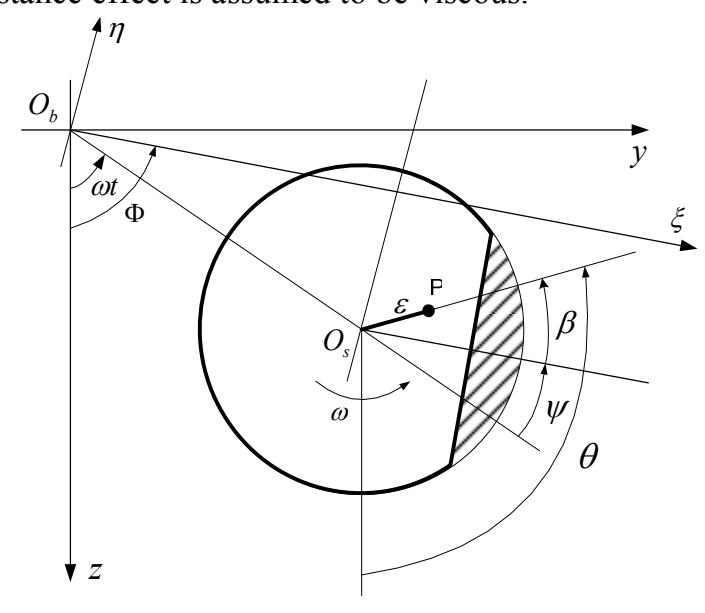

Figure 1. Section view of cracked Jeffcot rotor.

The angular position of the unbalance vector can be expressed as $\theta(t)=\omega t+\psi(t)+\beta$, where $\omega$ is a constant spin speed of the shaft, $\psi(t)$ is a torsional angle, and $\beta$ is the fixed angle between the unbalance vector and the centerline of the transverse shaft surface crack. It should be noted that

$$
\Phi(t)=\omega t+\psi(t), \dot{\theta} \equiv \dot{\Phi}=\omega+\dot{\psi} \text {, and } \ddot{\theta}=\ddot{\Phi}=\ddot{\psi}
$$

where $\Phi$ is the spin angle of the rotor.

The kinetic and potential energy for the rotor system subjected to lateral and torsional vibrations can be expressed as follows:

$$
\begin{gathered}
T=\frac{1}{2} J_{p} \dot{\theta}^{2}+\frac{1}{2} M\left(\dot{z}^{2}+\dot{y}^{2}\right)+\frac{1}{2} M \varepsilon^{2} \dot{\theta}^{2}+M \varepsilon \dot{\theta}(-\dot{z} \sin \theta+\dot{y} \cos \theta) \\
U=\frac{1}{2}\left\{\begin{array}{ll}
z & y
\end{array}\right\} \mathbf{K}_{I}\left\{\begin{array}{l}
z \\
y
\end{array}\right\}+\frac{1}{2} k_{t} \psi^{2}
\end{gathered}
$$

where

$$
\mathbf{K}_{I}=\left[\begin{array}{ll}
k_{z z} & k_{z y} \\
k_{y z} & k_{y y}
\end{array}\right]
$$

is the shaft stiffness matrix in inertial coordinates, with the off-diagonal elements corresponding to the asymmetry introduced either by a crack. Using the Lagrange approach, the nonlinear, coupled equations for transverse and torsional motion for the rotor system take the following form:

$$
\begin{gathered}
M \ddot{z}+C_{l} \dot{z}+k_{z z} z+k_{z y} y=F_{z}+M \varepsilon\left(\dot{\theta}^{2} \cos \theta+\ddot{\theta} \sin \theta\right) \\
M \ddot{y}+C_{l} \dot{y}+k_{y z} z+k_{y y} y=F_{y}+M \varepsilon\left(\dot{\theta}^{2} \sin \theta-\ddot{\theta} \cos \theta\right) \\
J_{p} \ddot{\psi}+C_{t} \dot{\psi}+\varepsilon C_{l}(\dot{z} \sin \theta-\dot{y} \cos \theta)-\varepsilon\left(F_{z} \sin \theta-F_{y} \cos \theta\right)+\varepsilon\left[\left(k_{z z} z+k_{z y} y\right) \sin \theta-\left(k_{y z} z+k_{y y} y\right) \cos \theta\right]+\frac{\partial U}{\partial \psi}=T_{e}(t)
\end{gathered}
$$


where $F_{\mathrm{z}}$ and $F_{\mathrm{y}}$ are the external forces (including gravity) in $z$ and $y$ directions, respectively, $C_{t}$ is torsional damping coefficient and $T_{e}$ is the externally applied torque.

\section{Uncracked shaft}

For the uncracked shaft the following conditions related to potential energy and stiffness components are satisfied:

$$
\frac{\partial U}{\partial \psi}=k_{t} \psi, k_{z z}=k_{y y} \equiv k \text { and } k_{z y}=k_{y z} \equiv 0
$$

\section{Cracked shaft}

The stiffness matrix for a Jeffcott rotor with a cracked shaft in rotating coordinates can be written as:

$$
\mathbf{K}_{R} \equiv\left(\begin{array}{cc}
k_{\xi} & 0 \\
0 & k_{\eta}
\end{array}\right)=\left(\begin{array}{cc}
k & 0 \\
0 & k
\end{array}\right)-f(\Phi)\left(\begin{array}{cc}
\Delta k_{\xi} & 0 \\
0 & \Delta k_{\eta}
\end{array}\right)
$$

where the first matrix refers to the stiffness of the uncracked shaft, and the second defines the variations in shaft stiffness $\Delta k_{\xi}$ and $\Delta k_{\eta}$ in $\xi$ and $\eta$ directions, respectively. The function $f(\Phi)$ is a crack steering function which depends on the angular position of the crack, $\Phi$, and the selected crack model.

The simplest crack model is the hinge model, where the crack is assumed to change from its closed to open state suddenly as the shaft rotates. The steering function for this model is defined as:

$$
f(\Phi)=\left\{\begin{array}{lll}
0 & \text { for } & \xi<0 \\
1 & \text { for } & \xi \geq 0
\end{array}\right.
$$

While the hinge model might be an appropriate representation for very small cracks, Mayes and Davies [8] proposed a model with a smooth transition between the opening and closing of the crack that is more adequate for larger cracks. In this case the crack steering function, or the Mayes modified function, takes the following form:

$$
f(\Phi)=\frac{1+\cos (\Phi)}{2}
$$

The stiffness matrix for a Jeffcott rotor with a cracked shaft in inertial coordinates, $\mathbf{K}_{I}$ is derived as

$$
\mathbf{K}_{I}=\mathbf{T K}_{R} \mathbf{T}^{-1}=\left(\begin{array}{ll}
k_{z z} & k_{z y} \\
k_{y z} & k_{y y}
\end{array}\right)=\left(\begin{array}{ll}
k & 0 \\
0 & k
\end{array}\right)-\frac{f(\Phi) k}{2}\left(\begin{array}{cc}
\Delta k_{1}+\Delta k_{2} \cos 2 \Phi & \Delta k_{2} \sin 2 \Phi \\
\Delta k_{2} \sin 2 \Phi & \Delta k_{1}-\Delta k_{2} \cos 2 \Phi
\end{array}\right)
$$

where the transformation matrix $\mathbf{T}$ is

$$
\mathbf{T}=\left(\begin{array}{cc}
\cos \Phi & -\sin \Phi \\
\sin \Phi & \cos \Phi
\end{array}\right)
$$

and

$$
\Delta k_{1}=\frac{\Delta k_{\xi}+\Delta k_{\eta}}{k} \text { and } \Delta k_{2}=\frac{\Delta k_{\xi}-\Delta k_{\eta}}{k}
$$

The cross-stiffness for deep cracks has been accounted for assuming $\Delta k_{\eta}=\Delta k_{\xi} / 6$, which based on relationship in Eq. (12), yields $\Delta k_{1}=\frac{7}{6} \frac{\Delta k_{\xi}}{k}$ and $\Delta k_{2}=\frac{5}{6} \frac{\Delta k_{\xi}}{k}$. The derivative of the potential energy term $\frac{\partial U}{\partial \psi}$ in Eq. (5) takes the following form: 


$$
\begin{aligned}
\frac{\partial U}{\partial \psi}= & -\frac{k}{4} \frac{\partial f(\Phi)}{\partial \psi}\left[\left(\Delta k_{1}+\Delta k_{2} \cos 2 \Phi\right) z^{2}+2 z y \Delta k_{2} \sin 2 \Phi+\left(\Delta k_{1}-\Delta k_{2} \cos 2 \Phi\right) y^{2}\right] \\
& +\frac{f(\Phi) k \Delta k_{2}}{2}\left[z^{2} \sin 2 \Phi-2 z y \cos 2 \Phi-y^{2} \sin 2 \Phi\right]+k_{t} \psi
\end{aligned}
$$

\section{RESULTS AND DISCUSSION}

A steel simple supported shaft with a disc at the center is considered. A transverse crack is located near the disk. The first lateral critical speed is $240 \mathrm{rad} / \mathrm{s}$. The parameters are shown in the Table 1.

Table 1. Parameters for a simple-supported Jeffcott rotor with a crack.

\begin{tabular}{ll}
\hline Disk mass, $M$ & $3 \mathrm{Kg}$ \\
Disk polar moment of inertia, $J_{p}$ & $0.01 \mathrm{~kg} \cdot \mathrm{m}^{2}$ \\
Eccentricity of the disk, $\varepsilon$ & $2.2 \times 10^{-5} \mathrm{~m}$ \\
Shaft stiffness, $k$ & $1.728 \times 10^{5} \mathrm{~N} / \mathrm{m}$ \\
Damping ratio in lateral direction, $\xi_{l}$ & \\
Damping ratio in torsional direction, $\xi_{t}$ & $0.075 \xi_{l}$ \\
Torsional natural frequency, $\omega_{t}$ & $600 \mathrm{rad} / \mathrm{s}$
\end{tabular}

The equations of motion (Eqs. (4)-(5)) were numerically integrated using Runge-Kutta method with the time integration step of $2 \pi /(360 \omega)$. This time step was small enough to get an accurate solutions even for slow rotational speeds such as $25 \mathrm{rad} / \mathrm{s}\left(\Delta t=6.9 \times 10^{-4} \mathrm{~s}\right)$. Bifurcation diagrams and power spectra for both lateral and torsional responses were used for presentation and analysis of the nonlinear system dynamical behavior. The bifurcation diagram plots the rotor orbit's $z$ - (or $\psi$-) coordinate (with a dot) for each shaft revolution as the keyphasor reference mark fixed on the rotor passes the same rotational angle. If the orbital motion were strictly synchronous, only the same "dot" would appear repeatedly.

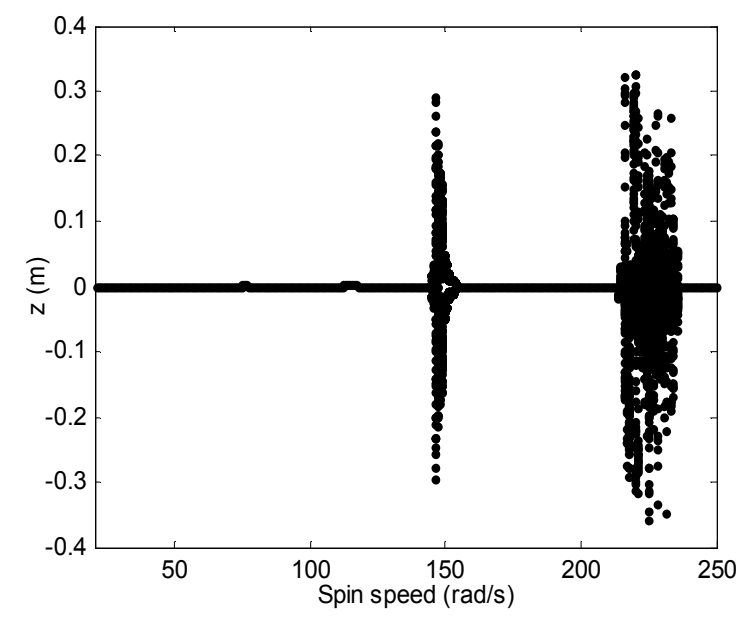

(a)

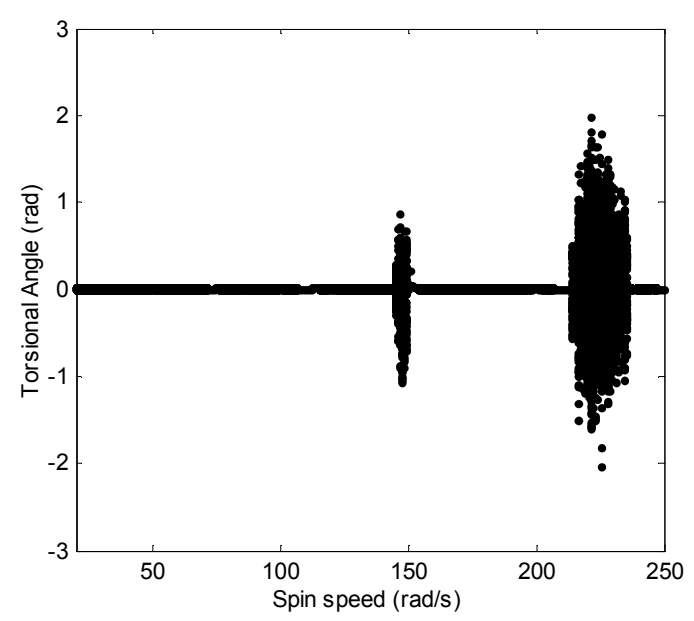

(b)

Figure 2. Bifurcation plots for cracked rotor: $\xi_{l}=0.008, \xi_{\mathrm{t}}=0.0006, \Delta \mathrm{k}_{\xi} / \mathrm{k}=0.4 ;$ (a) lateral response; (b) torsional response.

Two bifurcation diagrams are presented in Figure 2, showing the calculated vertical and angular rotor displacements at each speed increment over the speed range from 5 to $260 \mathrm{rad} / \mathrm{s}$, for the cracked rotor model with the crack's depth of 
0.4 , and lateral and torsional damping ratios $\xi_{l}=0.008$ and $\xi_{t}=0.0006$, respectively. The bifurcation plots appear to be alike; the results for the two different vibrational responses are comparable in terms of their general shapes. Resonances occur just below the lateral natural frequency and slightly above the half of lateral natural frequency, which one would expect for a non-linear system of this kind. Spanning the rotor spin speed in the range of 145-149 rad/sec and $216-234 \mathrm{rad} / \mathrm{sec}$, reveals the presence of a wide variety of the excited response characteristics. This is demonstrated in Figs. 3 and 4, showing the magnified "snapshots" of the sections of bifurcation diagram presented in Figure 2(a), and the corresponding orbital motions. During the chaotic behavior the lateral vibration amplitudes increase more than 1000 times, which would be equivalent to the system failure. Figure 2(b) shows torsional response of the rotor which is not subjected to any externally applied torque excitation, and provides clear evidence for the existence of lateral/torsional coupling in the system due to presence of lateral excitations and crack.
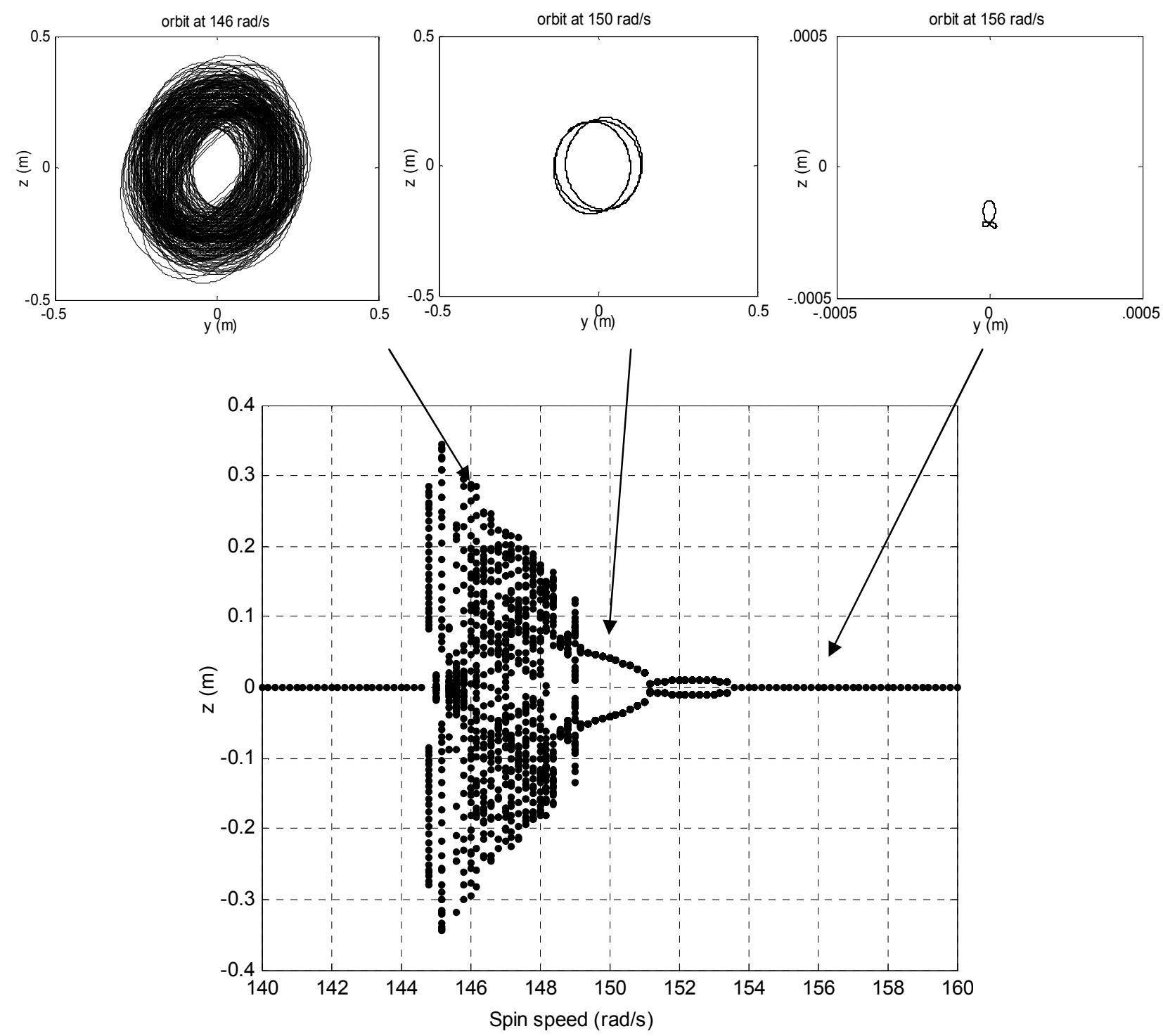

Figure 3. Bifurcation plot for the cracked rotor: $\xi_{l}=0.008, \xi_{\mathrm{t}}=0.0006, \Delta \mathrm{k}_{\xi} / \mathbf{k}=0.4$. 

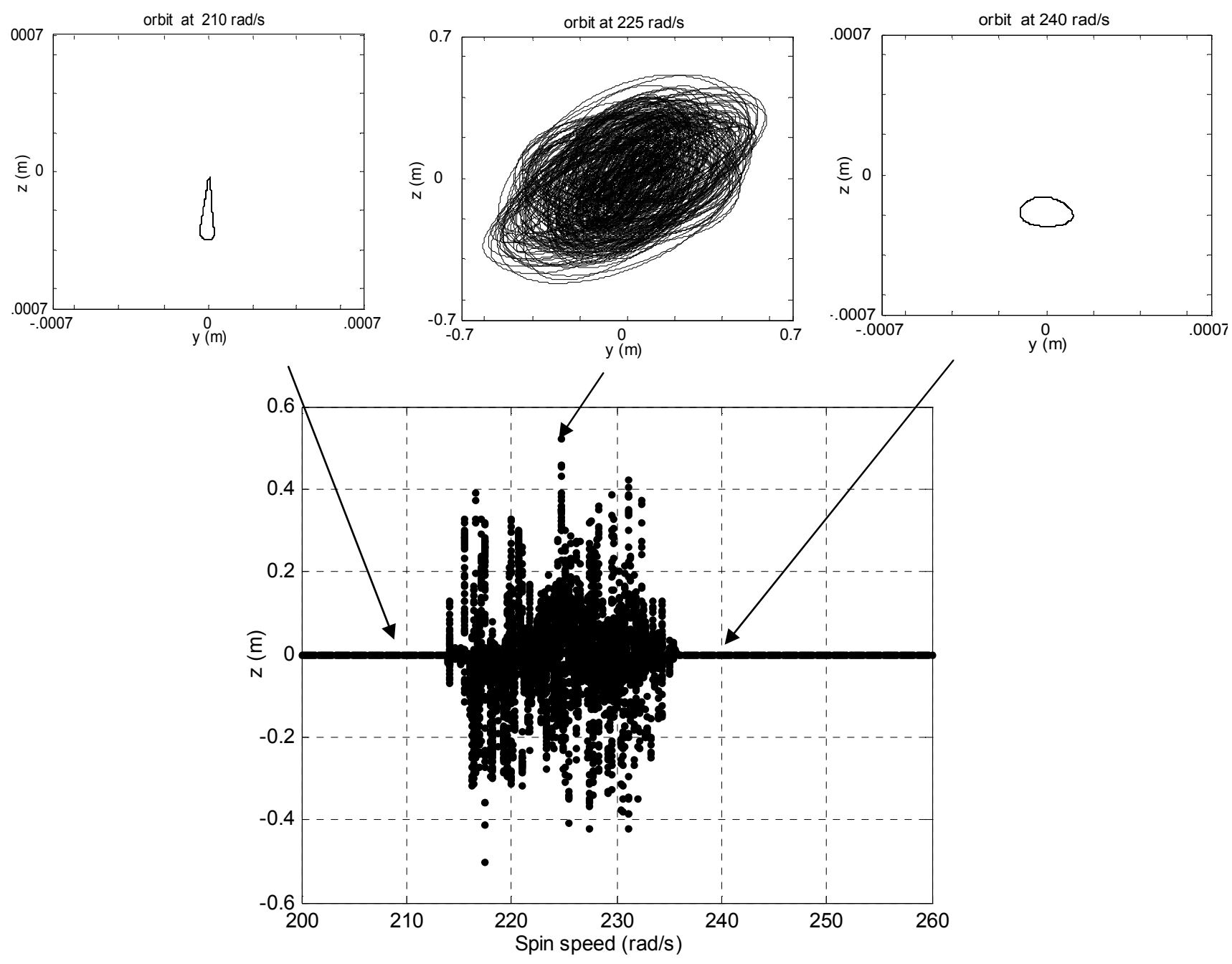

Figure 4. Bifurcation plot for the cracked rotor: $\xi_{l}=0.008, \xi_{\mathrm{t}}=0.0006, \Delta \mathrm{k}_{\xi} / \mathrm{k}=0.4$.

Figure 5 illustrates the lateral vibration responses for the uncracked and cracked rotor running at speed of $225 \mathrm{rad} / \mathrm{s}$. There is no external torsional excitation applied to the rotor. It can be seen that uncracked rotor vibrates around its static deflection position with the small vibration amplitude (Fig. 5(a)). The corresponding power spectrum shows only synchronous frequency component due to unbalance excitation. In the presence of transverse crack of depth of 0.4 , the rotor vibration amplitudes increase significantly, and the power spectrum illustrates the apparent system nonlinearity, indicating the multi-frequency content present in the vibration signal (Fig. 5(b)).

Note the window of the FFT is taken to be the last steady state 50 system rotations. This condition was reached by allowing the inherent system hysteresis damp out all starting effects in the first 200 revolutions. If the motion is periodic or quasi-periodic, then the power spectrum will consist of a sequence of spikes at the fundamental frequencies, their harmonics, and the frequencies that are the sums and differences of the various frequencies. For the chaotic motion (e.g., non-periodic but non-random), the power spectrum shows a random broadband character.

Figure 6 shows the torsional vibration responses for the uncracked and cracked rotor running at speed of $225 \mathrm{rad} / \mathrm{s}$., without external torsional excitation applied to the rotor. Due to the nonlinear lateral/torsional coupling the unbalance excitation induces the uncracked rotor torsional response at predominantly torsional resonance frequency $(600 \mathrm{rad} / \mathrm{sec})$ and superharmonic frequency $(2 \omega)$, (see Fig. 6(a)). After introducing transverse crack, the nonlinearities caused by the 
breathing of the crack strengthen the modes coupling. There exist many frequencies in the torsional frequency domain. Unbalance excitation not only causes the amplitude of torsional vibration to increase $10^{3}$ times larger than that without crack, but also significantly change the vibration patterns (Fig. 6 (b)).
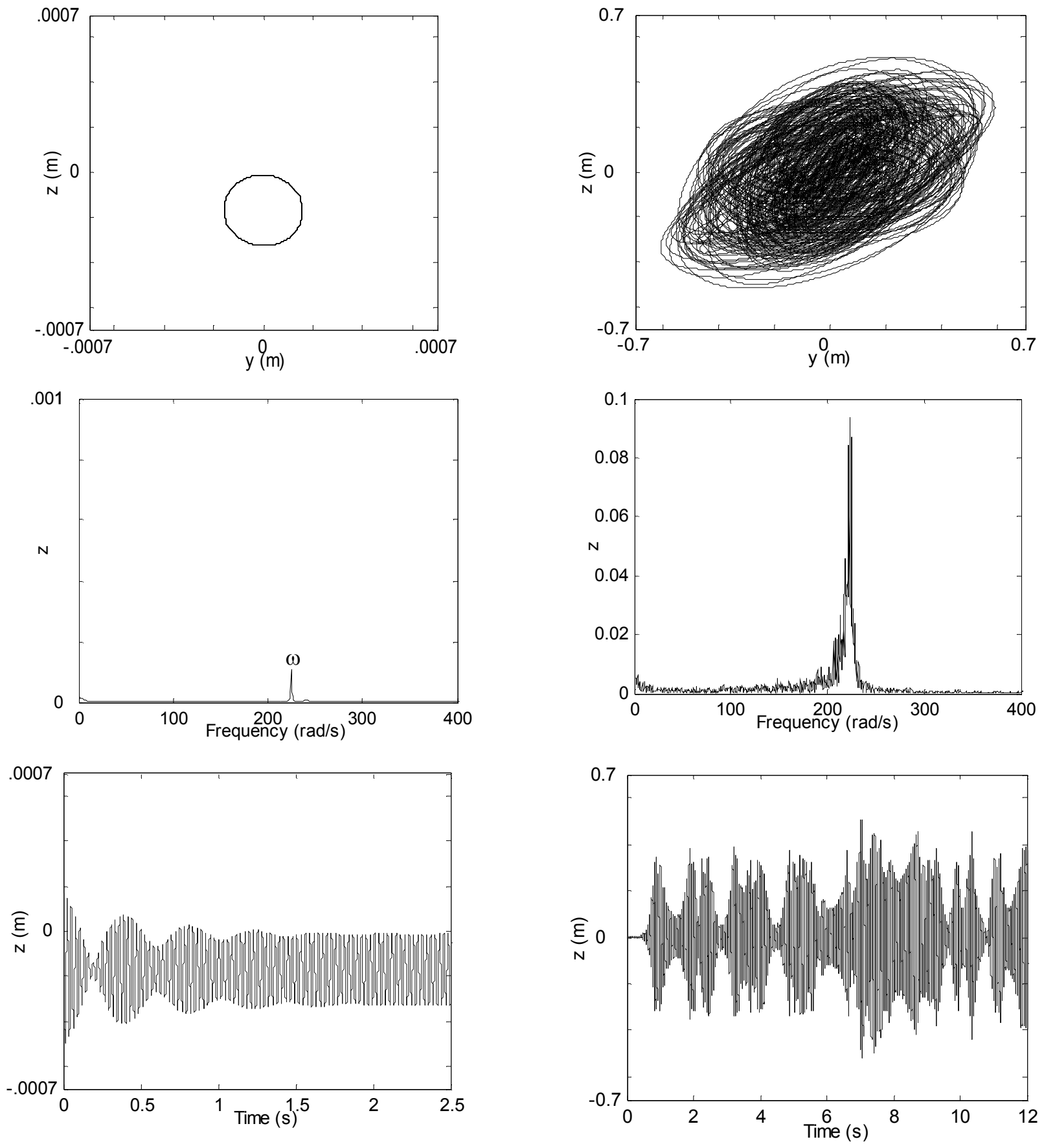

(a)

(b)

Figure 5. Lateral vibration response of rotor at speed $225 \mathrm{rad} / \mathrm{s}, \xi_{l}=0.008, \xi_{\mathrm{t}}=0.0006$; (a) without crack; (b) with crack $\Delta \mathbf{k}_{\xi} / \mathbf{k}=0.4$. 

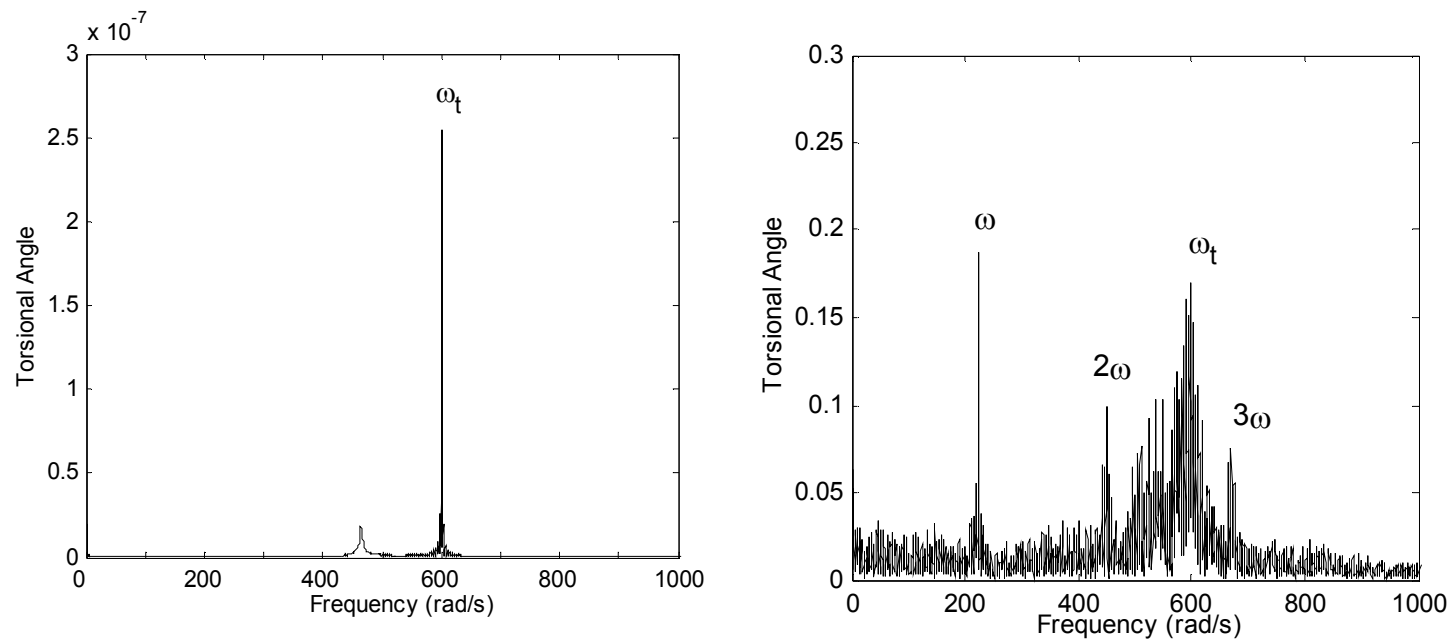

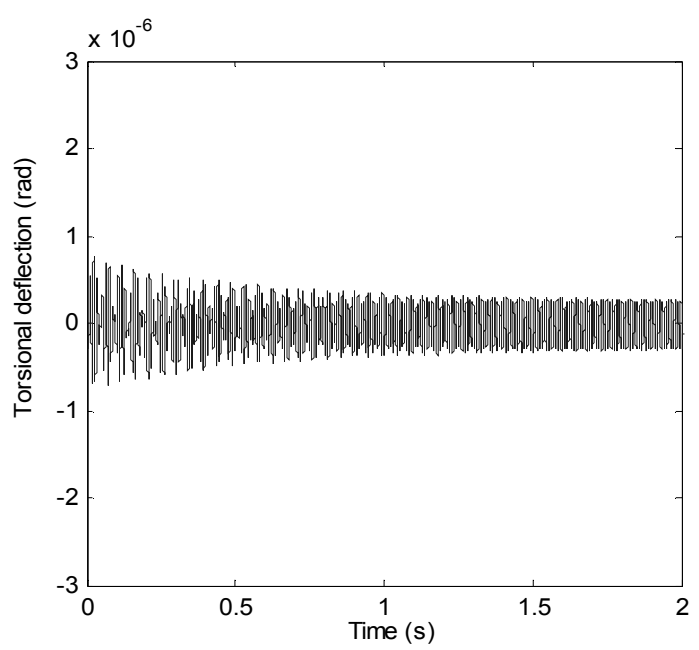

(a)

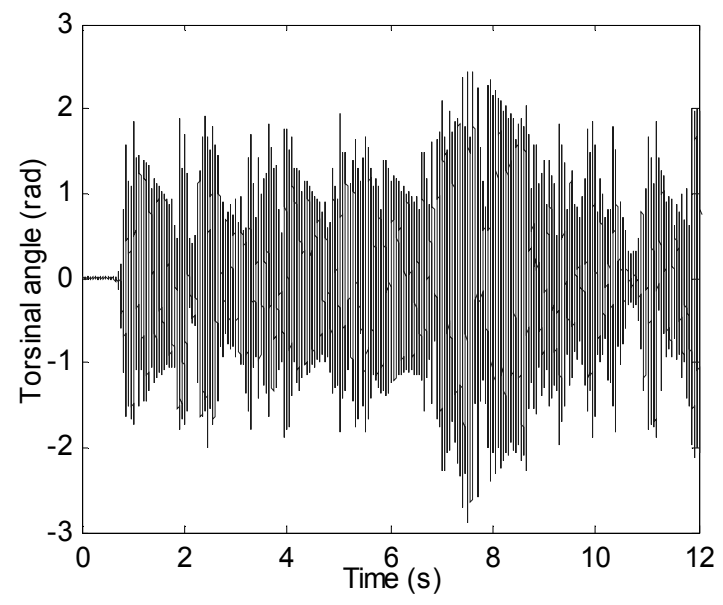

(b)

Figure 6. Torsional vibration response of rotor at speed $225 \mathrm{rad} / \mathrm{s}, \xi_{l}=0.008, \xi_{\mathrm{t}}=0.0006$; (a) without crack; (b) with crack $\Delta \mathbf{k}_{\xi} / \mathbf{k}=0.4$.

The comparison of lateral and torsional response for the cracked rotor is presented in Figure 7. Here, the values of parameters used for simulation are the same as those used for Figures 5 and 6, except for the increased value of damping, i.e., $\xi_{l}=0.048$ and $\xi_{t}=0.075 \xi_{l}$. By comparing responses shown in Figs. 5(b) and 6(b) with the ones on Fig. 7, one can observe that the chaotic behavior of the response disappear for this values of the increased damping.

Figures 8 and 9 illustrate bifurcation diagrams showing the response for different damping levels $\left(\xi_{l}=0.005-0.05\right)$ and depth of the crack ( $\Delta k_{\xi} / k=0.2-0.4$ ), respectively. These results clearly suggest the possibility of the use of chaos mapping of monitored vibration signals as a diagnostic tool to detect some rotating machinery malfunctions. As the damping ratio or crack depth are ranged over the interval illustrated in Figs. 8 and 9, a wide variety of response characteristics are excited.

Finally, a torsional excitation of $T_{e}(t)=800 \sin (240 t)$ was applied to the case of the cracked rotor, again, in addition to the unbalance and gravity forces. These results are shown in Fig. 10. The selected rotor spin speeds are an integer and not-integer fraction of the bending natural frequency, i.e., $0.1 \omega_{n}=24 \mathrm{rad} / \mathrm{s}$ and $0.13 \omega_{n}=31 \mathrm{rad} / \mathrm{s}$, respectively. For both speed cases the power spectra look similar, in addition to such crack "indicators" as the $2 \mathrm{X}$ and $3 \mathrm{X}$ components, the 

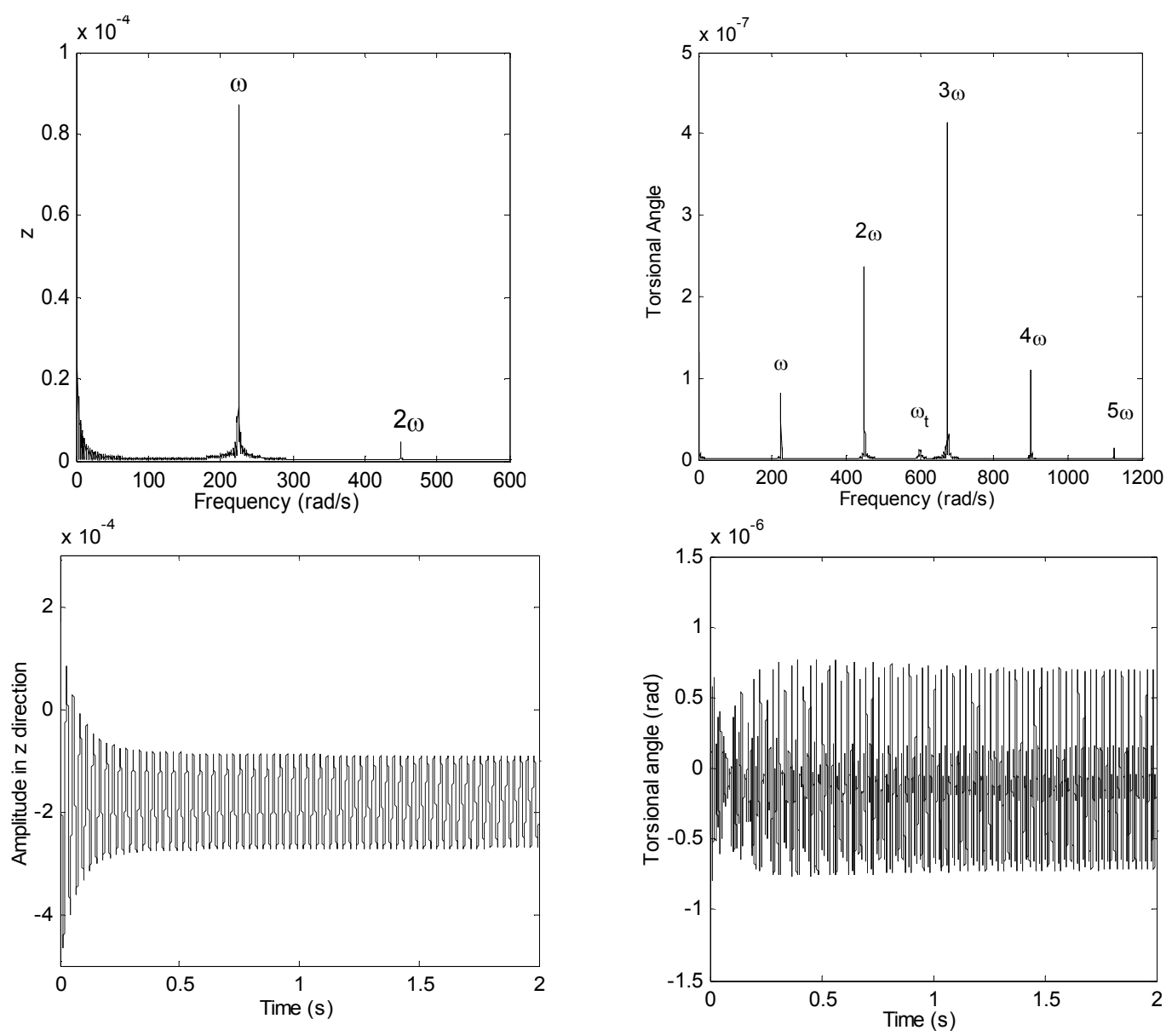

(a)

(b)

Figure 7. Vibration of cracked rotor at speed $225 \mathrm{rad} / \mathrm{s} ; \xi_{l}=0.048, \xi_{\mathrm{t}}=0.075 \xi_{l}, \Delta \mathrm{k}_{\xi} / \mathrm{k}=0.4$; (a) lateral response; (b) torsional response.

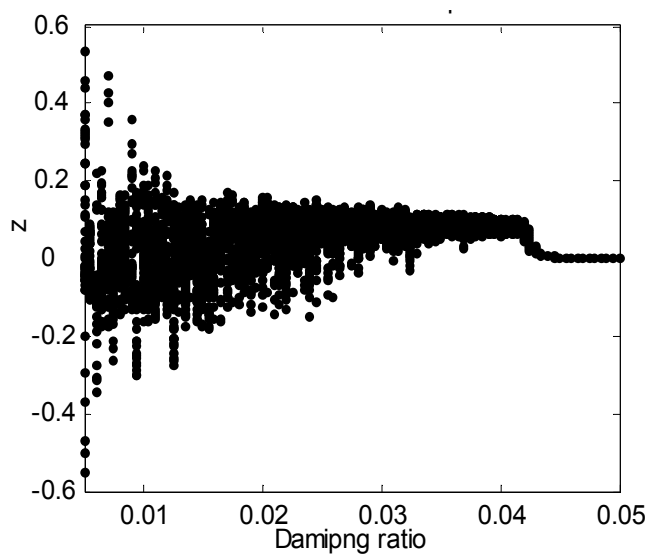

(a)

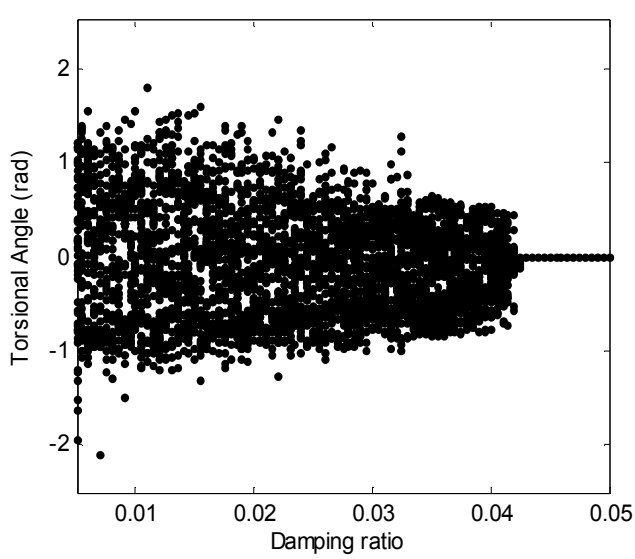

(b)

Figure 8. Bifurcation plots for cracked rotor at speed $\omega=225 \mathrm{rad} / \mathrm{s} ; \xi_{t}=0.075 \xi_{l}, \Delta \mathbf{k}_{\xi} / \mathbf{k}=0.4 ;$ (a) lateral response; (b) torsional response. 


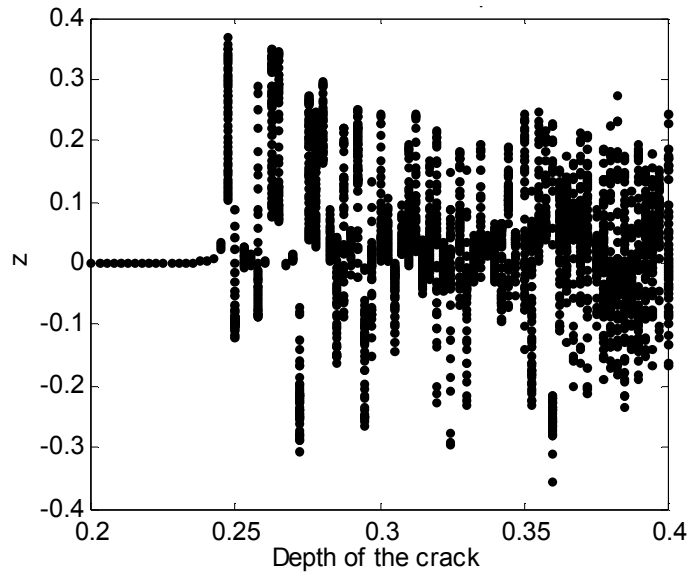

(a)

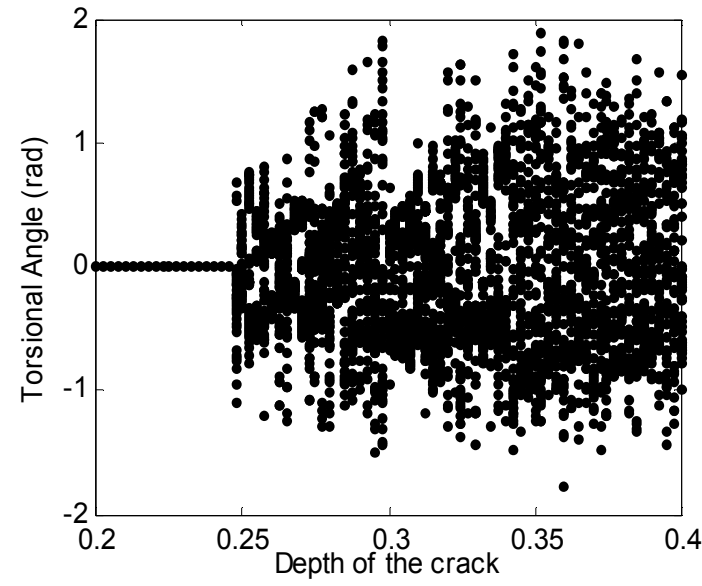

(b)

Figure 9. Bifurcation plots for cracked rotor at speed $\omega=225 \mathrm{rad} / \mathrm{s}, \xi_{l}=0.01 ; \xi_{t}=0.075 \xi_{l}, \Delta \mathrm{k}_{\xi} / \mathbf{k}=0.4 ;$ (a) lateral response; (b) torsional response.
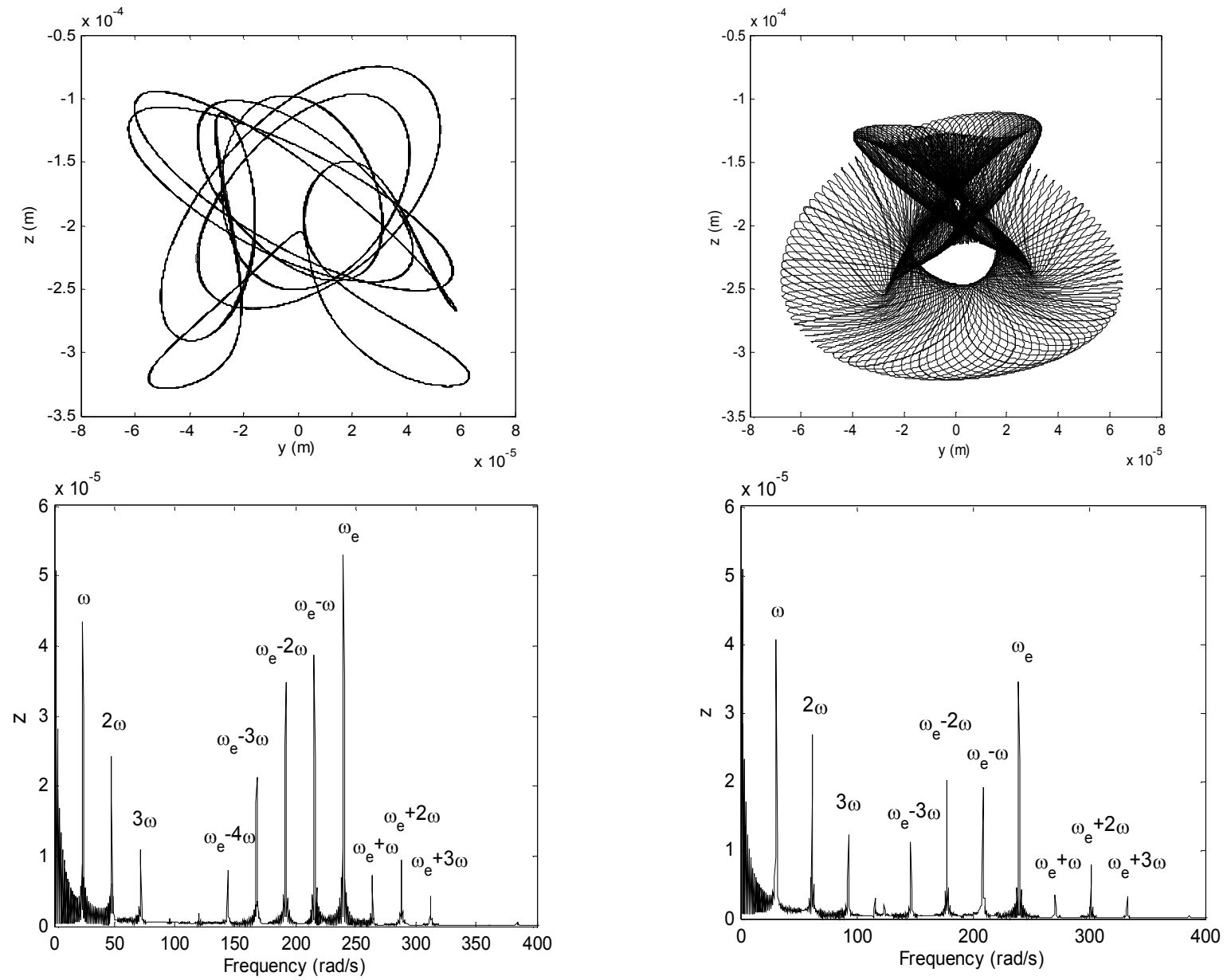

(a)

(b)

Figure 10. Orbits and power spectra of cracked rotor; $\xi_{l}=0.008, \xi_{t}=0.075 \xi_{l}, \Delta \mathbf{k}_{\xi} / \mathbf{k}=0.4$, $T_{\mathrm{e}}(t)=800 \sin (240 t)$; (a) speed $24 \mathrm{rad} / \mathrm{s} ;$ (b) speed $31 \mathrm{rad} / \mathrm{s}$. 
torsional excitation frequency $\omega_{e}=240 \mathrm{rad} / \mathrm{sec}$ is seen along with the side frequencies $\omega_{e} \pm \omega, \omega_{e} \pm 2 \omega$, and $\omega_{e} \pm 3 \omega$, which are located around $\omega_{e}$. These frequencies are generated as a result of the non-linear mechanism of the breathing crack under the influence of unbalance, gravity, and harmonic torsional excitation. However, the analysis of the orbital motion reveals that in the case of speed of $31 \mathrm{rad} / \mathrm{s}$ the orbit rotates in the opposite direction of the rotor's rotating direction. Simulations of orbits considering frequencies shown in corresponding to this case FFT spectrum (see Fig. 10(b)) have shown presence of frequency components being backward whirl fraction. Therefore, for this speed, the rotor vibration in the presence of the applied external torque is not periodic but quasi-periodic, where the ratios of the involved frequencies are not the ratios of integers.

\section{CONCLUSIONS}

An analytical solution was developed for the Jeffcott rotor taking into account coupling between the torsional and bending vibrations. This was achieved by the inclusion of the third equation for torsional vibration. The modeling was further enhanced by considering the nonlinearities associated with a breathing crack located on the shaft adjacent to the disk. Numerical simulations of the uncracked and cracked cases were carried out by applying an unbalance excitation, gravity forces, and a torsional excitation.

When comparing the torsional and lateral frequency spectrums for the uncracked and cracked cases, very clear distinctions were noticed. In each case (uncracked and cracked), the system was excited by the residual unbalance and gravitational forces, and then by the addition of a torsional excitation. The sum and difference frequencies have been observed in a response of cracked rotor due to unbalance and external torsional excitation. By employing nonlinear vibration theory, some phenomena evolving out of crack in rotating systems have been addressed, such as the numerical study of the bifurcation of the parameter dependent system.

The uniqueness of the observed crack signatures, captured by the analytical approach, demonstrates an improvement in early diagnosis of rotor cracks.

\section{NOMENCLATURE}

$C_{l} \quad$ Damping coefficients in $\mathrm{z}$ and $\mathrm{y}$ direction, respectively

$C_{l} \quad$ Torsional damping coefficient

$F_{z}, F_{y} \quad$ External forces in $z$ and $y$ directions, respectively

$J_{p} \quad$ Disk polar moment of inertia

$M \quad$ Disk mass

$\omega \quad$ Constant spin speed of the shaft

$\psi \quad$ Torsional angle

$\beta \quad$ Angle between the unbalance vector and the crack center line

$k \quad$ Stiffness of uncracked rotor.

$k_{z z}, k_{y y} \quad$ Direct stiffness terms in $z$ and $y$ direction, respectively.

$k_{z y} \quad$ Cross stiffness term introduced by the shaft asymmetry

$k_{t} \quad$ Torsional stiffness

$\varepsilon \quad$ Eccentricity of the disk

$\xi_{l} \quad$ Damping ratio in lateral direction

$\xi_{t} \quad$ Damping ratio in torsional direction

$\omega_{t} \quad$ Torsional natural frequency

$\omega_{n} \quad$ Lateral natural frequency

$T_{e}(t) \quad$ External torque

$\zeta, \xi, \eta \quad$ Rotor-fixed coordinates system 
$\begin{array}{ll}z, y & \text { Inertia coordinate system } \\ \Delta k_{\xi}, \Delta k_{\eta} & \text { Reduced stiffness in } \xi \text { and } \eta \text { direction, respectively }\end{array}$

\section{REFERENCES}

1. Tondl, A., "Some Problems of Rotor Dynamics", Publishing House of Czechoslovakia Academy of Science, Praque, 1965.

2. Cohen, R. and Porat, I., "Coupled Torsional and Transverse Vibration of Unbalanced Rotor," ASME Journal of Applied Mechanics, Vol. 52, pp. 701-705, 1985.

3. Bernasconi, O., "Bisynchronous Torsional Vibrations in Rotating Shafts," ASME Journal of Applied Mechanics, Vol. 54, pp. 893-897, 1987.

4. Muszynska, A., Goldman, P. and Bently, D. E., "Torsional/Lateral Cross-Coupled Responses Due to Shaft Anisotropy: A New Tool in Shaft Crack Detection”, I. Mech. E., C432-090, Bath, United Kingdom, pp. 257-262, 1992.

5. Bently, D. E., Goldman, P. and Muszynska, A., ““'Snapping” Torsional Response of an Anisotropic Radially Loaded Rotor," Journal of Engineering for Gas Turbines and Power, Vol. 119, pp. 397-403, 1997.

6. Sawicki, J.T., Wu, X., Baaklini, G., and Gyekenyesi, A.L., "Vibration-Based Crack Diagnosis in Rotating Shafts During Acceleration through Resonance," Proceedings of SPIE $10^{\text {th }}$ Annual International Symposium on Smart Structures and Materials, San Diego, California, 2003.

7. Sawicki, J.T., Bently, D.E., Wu, X., Baaklini, G., and Friswell, M.I., "Dynamic Behavior of Cracked Flexible Rotor Subjected to Constant Driving Torque," Proceedings of the $2^{\text {nd }}$ International Symposium on Stability Control of Rotating Machinery, Gdansk, Poland, pp. 231-241, 2003.

8. Mayes, I. W. and Davies, W. G. R., "Analysis of the Response of a Multi-Rotor-Bearing System Containing a Transverse Crack in a Rotor", ASME Journal of Vibration, Acoustics, Stress, and Reliability in Design, Vol. 106, pp 139-145, 1984. 